\title{
PERFORMANCE OF A COINCIDENCE BASED BLOOD ACTIVITY MONITOR
}

\author{
William W. Moses, \\ Research Medicine and Radiation Biophysics Division, \\ Lawrence Berkeley Laboratory, \\ 1 Cyclotron Road, Berkeley, CA 94720
}

\section{Abstract}

A new device has been constructed that measures the positron emitting radio-tracer concentration in arterial blood by extracting blood with a peristaltic pump, then measuring the activity concentration by detecting coincident pairs of $511 \mathrm{keV}$ photons with a pair of heavy inorganic scintillators attached to photomultiplier tubes. The sensitivity of this device is experimentally determined to be 610 counts/second per $\mu \mathrm{Ci} / \mathrm{ml}$, and has a paralyzing dead time of $1.2 \mu \mathrm{s}$, so is capable of measuring blood activity concentrations as high as $1 \mathrm{mCi} / \mathrm{ml}$. Its performance is compared to two other blood monitoring methods: discrete blood samples counted with a well counter and a device that uses a plastic scintillator to directly detect positrons. The positron detection efficiency of this device for ${ }^{18} \mathrm{~F}$ is greater than the plastic scintillation counter, and also eliminates the radioisotope dependent correction factors necessary to convert count rate to absolute concentration. Coincident photon detection also has the potential of reducing the background compared to direct positron detection, thereby increasing the minimun detectable isotope concentration.

\section{Introduction}

In order to perform dynamic function studies with positron emission tomography (PET), it is necessary to accurately measure the radio-tracer concentration in arterial blood as a function of time. This concentration versus time, known as the arterial input function, is usually measured by extracting arterial blood and measuring the radio-tracer concentration with one of the following two methods. The first method is to periodically withdraw a number of discrete samples, then measure the activity of each individual sample in a well counter [1]. Although this method has high sensitivity (i.e. a large number of detected events for a given concentration), it requires a significant amount of sample handling and preparation time, and thus is both

- This work was supported in part by the U.S. Department of Energy under contract No. DE-AC03-76SF00098, and in part by Nocional Institute of Health Biomedical Research Support Grant No. RR05918.

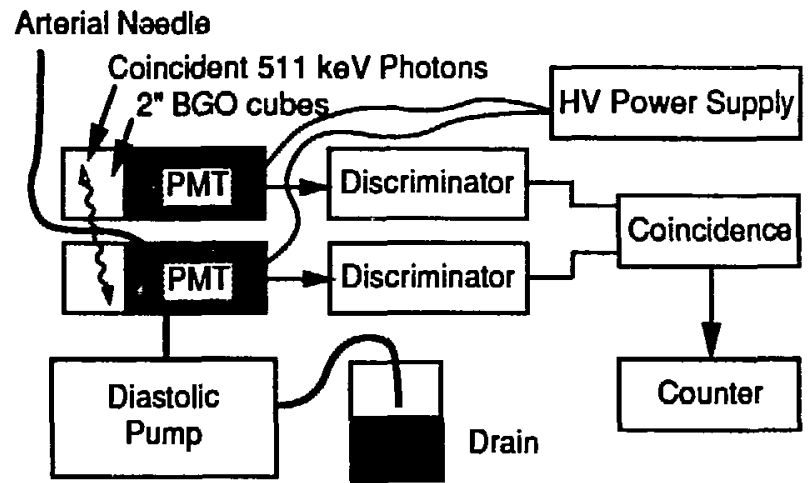

Figure 1: Sketch of the Blood Activity Monitor.

expensive and unsuitable for use with short-lived radiotracers such as ${ }^{15} \mathrm{O}$ and ${ }^{82} \mathrm{Rb}$. The second method is to continuously withdraw blood with a peristaltic pump, then monitor the blood as it is being withdrawn by directly detecting positrons with a plastic scintillator and a photomultiplier tube $[2,3,4]$.

This direct detection method eliminates the need to handle a large number of individual samples and is able to be used with short lived isotopes. However, most devices of this type surround the blood with a plastic tube, and so the emitted positron must pass through both the blood and the tubing wall before depositing energy in the plastic scintillator and being detected. Since the penetration ability depends on the positron energy and hence the tracer isotope, the detection efficiency for these devices is isotope dependent, necessitating an isotope dependent calibration factor to convert measured count rate into absolute activity. For example, the probability of penetrating the plastic tubing in the device described by Eriksson, et al. [4] (0.94 $\mathrm{mm}$ inside diameter, $1.22 \mathrm{~mm}$ outside diameter) is computed to be $85 \%$ for ${ }^{15} \mathrm{O}$, but only $33 \%$ for ${ }^{18} \mathrm{~F}$-FDG [5], resulting in a low sensitivity for this commonly used PET tracer. In addition, the counting efficiency depends strongly on the density of the material being sampled the counting rate for identical concentrations of ${ }^{11} \mathrm{C}$ in water and in blood differ by $10 \%$ [4]. Finally, the plastic scintillator is susceptible to backgrounds from low energy emissions from radioactive daughters and contaminants. 
For example, ${ }^{122}$ I usually contains a small contaminant of ${ }^{125}$ I that tends to adhere to the walls of the tube. This isotope emits a $35 \mathrm{keV}$ photon, which has a high probability of being absorbed by the plastic scintillator and incorrectly identified as positron emission from ${ }^{122} \mathrm{I}$.

While it has been suggested the sensitivity of direct positron detection devices can be improved by placing the blood in closer proximity to the plastic scintillator $[6,7]$, many of the above problems can be eliminated by detecting coincident pairs of $511 \mathrm{keV}$ photons resulting from positron annihilation rather than detecting positrons directly. Since $511 \mathrm{keV}$ photons easily penetrate both the blood and the tubing, the conversion factor from measured count rate to absolute concentration is independent of the thickness of tube wall and density of the material being sampled, and since this method is insensitive to the positron range, the same calibration can be used for all isotopes. In addition, backgrounds from radioactive daughters and contaminants are eliminated. Such a coincidence based blood sampling device has been reported on by the Karolinska Institute [4]. The device described herein differs in that it uses larger BGO crystals, thereby increasing the sensitivity by a factor of nearly three. In addition, faster coincidence electronics have decreased the dead time by a factor of twenty.

\section{Equipment}

A device has been constructed that replaces the plastic scintillator sampling system with two heavy atom scintillators, each individually coupled to a photomultiplier tube as shown in Figure 1. A pair of two inch cubes of bismuth germanate (BGO) scintillator are individually glued to Hamamatsu R329-02 photomultiplier tubes operated at $-1500 \mathrm{~V}$. A semi-circular slot is ground bisecting one face of each BGO crystal, and all exposed surfaces of the BGO crystals are coated with a reflective coating of Magnesium Oxide powder and wrapped with aluminum foil and black tape to block ambient light. The scintillator/photomultiplier tube assemblies are placed next to each other with the slots in the BGO aligned to form a $\frac{3}{16}$ "diameter hole to accommodate the blood carrying tube. A photograph of this assembly is shown in Figure 2.

The scintillator/photomultiplier tube assembly is surrounded on five sides with lead in order to reduce background from both the patient and the receptacle that collects the blood after its activity is measured. The lead is two inches thick on the two sides that face toward the patient, and one inch thick on the remaining three sides. The lead is surrounded on all six sides by a light tight box of $\frac{1}{16}$ " soft iron that is pierced by a straight length of $\frac{1}{16}$ " diameter $0.016^{\prime \prime}$ wall stainless steel tubing, as well as feedthroughs for the high voltage and photomultiplier tube outputs. The tube enters the steel box on the patient side, passes through the hole formed by the slots in the two scintillators, and exits through the opposite side of the box. This stainless steel tube is used as a guide

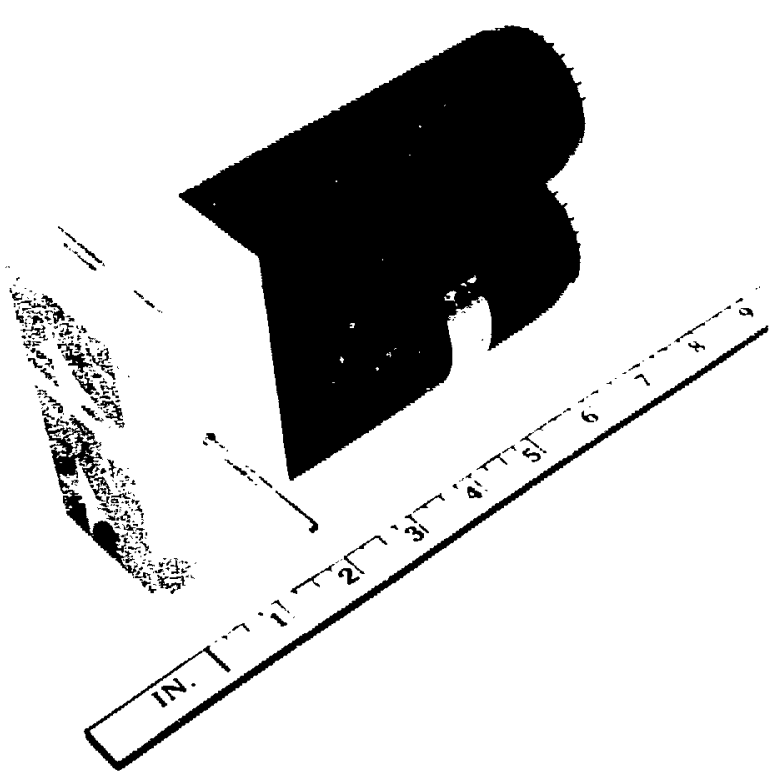

Figure 2: Scintillator/Photomultiplier Tube Assembly.

for the plastic tubing containing the blood from the peristaltic pump, and is attached to the steel box with black RTV cement to keep ambient light out of the box. The final assembly, which measures 5 inches by 6.5 inches by 12 inches and weighs approximately 70 pounds, is pictured in Figure 3.

The output of each photomultiplier tube is amplified and shaped with a $100 \mathrm{~ns}$ integration and $200 \mathrm{~ns}$ differentiation time constant, and this shaped signal is used to form a 500 ns wide timing signal with an Ortec $473 \mathrm{~A}$ constant fraction discriminator whose threshold is set at approximately $100 \mathrm{keV}$. Time coincidences are detected with an Ortec 418A universal coincidence module with a 100 ns acceptance window, then converted to NIM logic levels and counted with a Jorway 84 CAMAC scaler.

\section{Performance}

The timing resolution of the system is measured by injecting a small quantity of positron emitting isotope into the plastic tube, then using an Ortec 457 time to amplitude converter (TAC) to measure the difference in arrival time of the pulses from the two photomultiplier tubes. The resuiting distribution, shown in Figure 4, has a fuli width at half maximum (FWHM) of 9.1 ns and a full width at tenth maximum of $22.2 \mathrm{~ns}$, which demonstrates that the $100 \mathrm{~ns}$ coincidence window used by the system is adequate.

The dead time and efficiency are measured by injecting a $900 \mu \mathrm{Ci} / \mathrm{ml}$ concentration of ${ }^{68} \mathrm{Ga}$-EDTA into the plastic tube, then measuring the coincidence count rate as the ${ }^{68} \mathrm{Ga}$ decays to obtain the measured count rate as a function of isotope concentration. The resulting relation. 


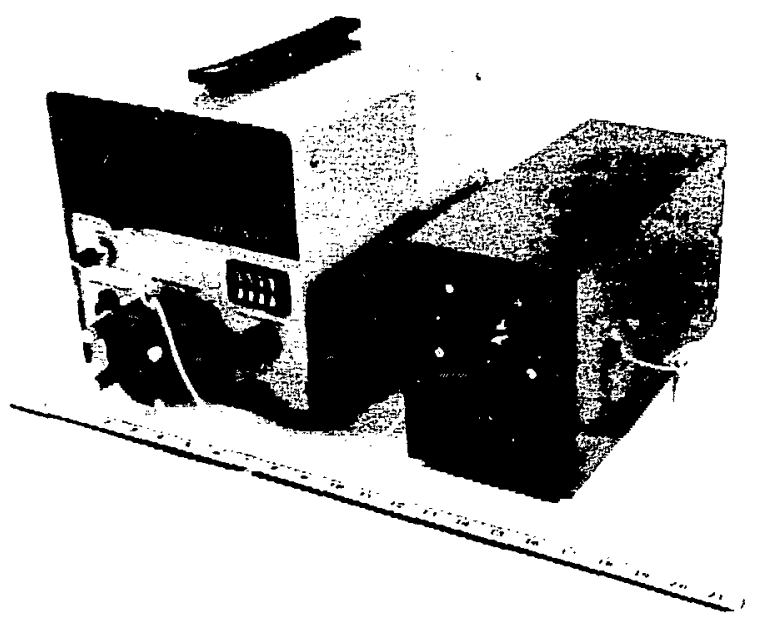

Figure 3: The Blood Activity Counter Assembly.

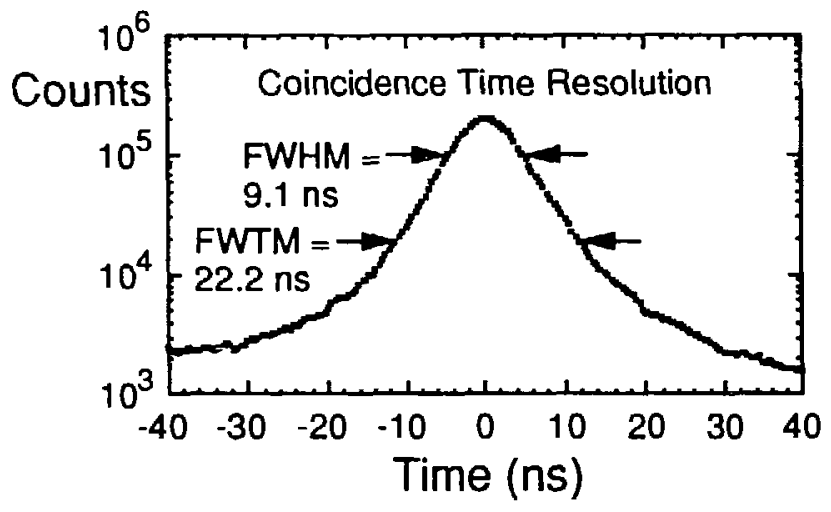

Figure 4: Coincidence Time Distribution

shown in Figure 5 , is well fit $\left(\chi^{2} / \mathrm{DOF}=0.98\right)$ by

$$
\text { Coincidence Rate }=610 * \mathcal{C} * e^{-1.2 \mu s * 610 * \mathcal{C}} \text {, }
$$

where $\mathcal{C}$ is the isotope concentration in $\mu \mathrm{Ci} / \mathrm{ml}$ (after correcting for the $90 \%$ positron emission probability of ${ }^{68} \mathrm{Ga}$ ) and the coincidence rate in measured in counts per second. Therefore this device has a sensitivity of 610 counts/second per $\mu \mathrm{Ci} / \mathrm{ml}$ which, since the volume of the tube surrounded by BGO is known $(0.044 \mathrm{ml})$, implies the detection efficiency for a positron emitted between the two crystals is $37.5 \%$. Equation 1 also states that the device is characterized by a paralyzing dead time of $1.2 \mu \mathrm{s}$, so the measured coincidence rate will increase with increasing isotope concentration until a concentration of $1.5 \mathrm{mCi} / \mathrm{ml}$. Therefore, this device is useful for measuring positron emitting isotope concentrations up to approximately $1 \mathrm{mCi} / \mathrm{ml}$.

The minimum detectable isotope concentration is defined as the concentration at which the coincidence rate due to positron annihilation detection is the same as the coincidence rate when zero activity is present in the device (i.e. the signal to noise ratio is $1: 1$ ). This device currently has an background coincidence rate of 1.5 counts

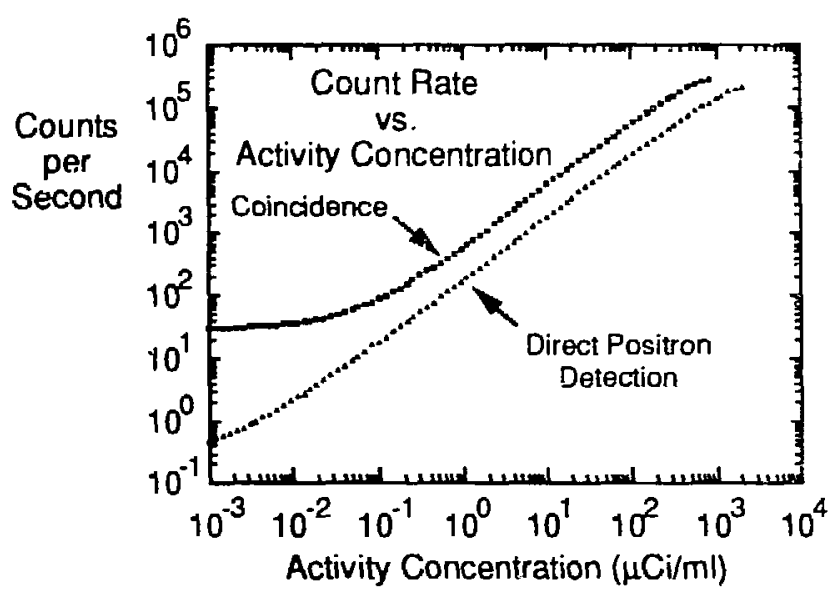

Figure 5: Count Rate vs. Activity Concentration.

per second, corresponding to a minimum detectable concentration of $2.4 \mathrm{nCi} / \mathrm{ml}$. However, the $\mathrm{BGO}$ crystals in this device are contaminated by small amount of ${ }^{68} \mathrm{Ge}$, a positron emitting isotope with a 288 day half-life. The accidental coincidence rate achievable after this contamination decays away is estimated by delaying one of the two timing signals with a $1 \mu \mathrm{s}$ cable. With this delay in place, a coincidence rate of 0.01 counts per second is measured, corresponding to a minimum detectable concentration of $16 \mathrm{pCi} / \mathrm{ml}$. Note that the data in Figure 5 shows a minimum coincidence rate of 30 counts/second. This is not due to accidental coinciciences or contamination of the detector, but contamination of the ${ }^{68} \mathrm{Ga}$-EDTA with ${ }^{68} \mathrm{Ge}$ due to breakthrough in the gallium generator column.

Attempts were made to reduce the accidental coincidence rate by imposing a tighter timing coincidence requirement, achieved by forming a timing signal from the unshaped photomultiplier tube output triggered at the single photoelectron level. This had little effect on the sensitivity, but increased the dead time to $13 \mu$ s because of the increased sensitivity to single photons. This increased sensitivity to single photons also caused the accidental coincidence rate to increase, thereby increasing the minimum detectable concentration.

The sensitivity of this coincidence based monitor has been compared to a direct $[$ asitron detection system. The device described by Eriksson, et al. [4] was duplicated, but with Teflon tubing $(0.042$ inch inside diameter, 0.066 inch outside diameter) in order to reduce blood clotting. By performing rate versus concentration measurements as before, a positron detection efficiency of $11.5 \%$ for ${ }^{18} \mathrm{~F}$-FDGi is measured for this direct detection system. The coincidence rate versus ${ }^{18} \mathrm{~F}$ concentration, again corrected for the $96.9 \%$ positron emission probability of ${ }^{18} \mathrm{~F}$, is also plotted in Figure 5. This efficiency is slightly less than the $18.5 \%$ positron detection efficiency computed using known positron range formulae [5], but is roughly half of the $33 \%$ efficiency predicted for the Karolinska device due to the increased tubing wall thickness. However, the coincidence 
based counter has an isotope indepencient $37 \%$ efficiency, and so is slightly more efficient that the Karolinska device and is three times more sensitive than our direct detection device for ${ }^{18} \mathrm{~F}$. Since the same length and diameter tubing is used in our direct positron detection and coincidence detection devices, this increase in sensitivity is due to improved detector response, not by an increased volume of material being sampled.

Three modalities of blood activity monitoring, individual samples measured in a well counter, the direct positron detertion monitor, and the coincidence based monitor, are conipared by measuring the blood activity concentration versus time in a dog. A $12 \mathrm{~kg}$ beagle was injected with $1.2 \mathrm{nCi}$ of ${ }^{18} \mathrm{~F}-\mathrm{FDG}$, and individual blood samples were withdiawn during a 40 minute period at intervals ranging from 10 seconds to 5 minutes. During this time arterial blood was also withdrawn with a peristaltic pump, the : ubing from the pump passing first through the direct detection monitor and then the coincidence based monitor. The three resulting time-activity curves are shown in Figure 6 .

The data in Figure 6 have been corrected for time offsets of approximately 10 seconds due to the time taken for the blood to travel down the tube, and since the samples were placed in the well counter several hours after they were drawn, the well counter rate plotted has been corrected for radioactive decay between the time the samples were drawn and they were counted. Care should be taken when using the well counter data to compare positron detection efficiencies, as the volume of each sample in the well counter was $1 \mathrm{ml}$, which is over twenty times larger than the volumed sampled by the coincidence or direct detection devices. The curves obtained with the direct detection method and the coincidence based method are very similar in shape, but the count rates measured with the coincidence detector are three times higher than they are for the direct positron detector. The shape of the individually sampled well counter data appears to differ slightly from the other two modalities, but this may be due to the fact that since it takes approximately 10 seconds to withdraw a sample for the well counter, these data are smeared by a 10 second time constant.

\section{Discussion and Conclusion}

In conclusion, a coincidence based blood activity moniter has been built, and found to have an isotope independent positron detection efficiency of $37.5 \%$. It is able to measure blood activity concentrations higher than normally encountered in PET studies $(1 \mathrm{mCl} / \mathrm{ml})$ without significant dead time or accidental coincidence correction. Its sensitivity is greater than devices that directly detect positron decay for positron emitting tracers with low end point energy, such as ${ }^{18} \mathrm{~F}$, yielding arterial input function data with less statistical noise for this commonly used PET tracer.

The capabilities of his device could be further improved

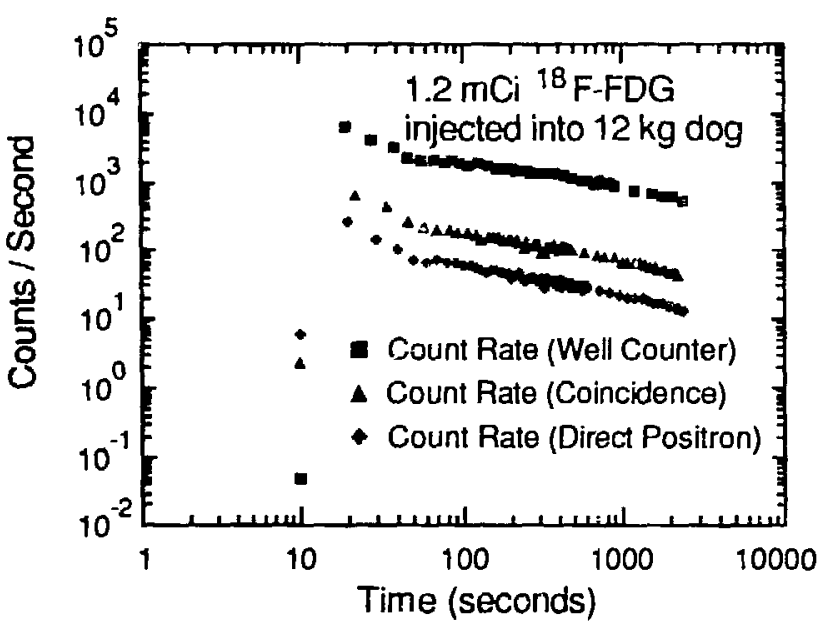

Figure 6: Count Rate vs. Time - Comparison of Three Blood Activity Monitoring Methods.

by replacing the scintillators with a faster heavy atoin scintillator, such as Cerium Fluoride [8,9] or Lead Carbonate [10]. Since the primary decay component of either of these two newly discovered scintillators is an order of magnitude faster than BGO ( $<30$ ns versus $300 \mathrm{~ns}$ ), replacing the BGO crystals with $\mathrm{CeF}_{3}$ or $\mathrm{PbCO}_{3}$ crystals will increase the maximum rate by an order of magnitude without sacrificing any other performance aspect. In addition, it would allow a storter coincidence timing window to be used, which would reduce the accidental coincidence rate and decrease the minimum detectable concentration.

\section{Acknowledgements}

I would like to thank Mr. John Cahoon and Mr. Tony Vuletich of Lawrence Berkeley Laboratory for technical assistance in building the coincidence based blood counter, Mr. Miguel Colina of Lawrence Berkeley Laboratory for assistance in data collection, Dr. Jan A. Litton of Karolinska Hospital and Mr. Miguel Colina, Dr. Chester A. Mathis, Dr. Peter E. Valk, and Dr. William A. Jagust of Lawrence Berkeley Laboratory for providing the direct positron detecting blood counter, and Dr. Kathleen Brennan and Mr. Craig Stalnaker for performing the dog surgery. This work was supported ini part by the Director, Office of Energy Research, Office of Health and Environmental Research of the U.S. Department of Energy, under contract No. DE-AC0376SF00098, and in part by National Institute of Health Biomedical Research Support Grant No. RR05918.

Reference to a company or product name does not imply approval or recommendation of the product by the University of California or the U.S. Department of Energy to the exclusion of others that may be suitable. 


\section{References}

[1] M.M. Graham and B.L. Lewellen. High speed automated discrete blood sampling for PET. In The Journal of Nuclear Medicine, Proceedings of the $35^{\text {th }}$ Annual Meeting, page 879, The Society of Nuclear Medicine, 1988.

[2] G.D. Hutchins, R.D. Hichwa, and R.A. Koeppe. A continuous flow input function detector for ${ }^{15} \mathrm{O}-\mathrm{H}_{2} \mathrm{O}$ blood flow studies in positron emission tomography. IEEE Trans. Nucl. Sci. NS-33, 546-549 (1986).

[3] H. Iida, I. Kanno, S. Miura, M. Murakami, K. Takahashi, and $\mathrm{K}$. Uemura. Error analysis of a quantitative cerebral blood flow measurement using ${ }^{15} \mathrm{O}_{-} \mathrm{H}_{2} \mathrm{O}$ autoradiography and positron emission tomography, with respect to the dispersion of the input function. J. Cereb. Blood Flow Metabol. 6, 536-545 (1986).

[1] L. Eriksson, S. Holte, C. Bohm, M. Kesselberg, and B. Hovander. Automated blood sampling systems for positron emission tomography. IEEE Trans. Nucl. Sci. NS-35, 703-707 (1988).

[5] S.E. Derenzo. Mathematical removal of positron range blurring in high resolution tomography. IEEE Trans. Nucl. Sci. NS-33, 565-569 (1986).

[6] J.J. Sunderland, C.C. Martin, S.B. Perlman, R.L. Levine, and R.J. Nickles. A high efficiency, high count rate capability automated blood sampler. In The Journal of Nuclear Medicine, Proceedings of the $36^{\text {th }}$ Annual Meeting, page 745, The Society of Nuclear Medicine, 1989.

[7] J.E. Litton and J. Eriksson. A detector for transcutaneous measurement of the arterial input function in positron emission tomography. IEEE Trans. Nucl. Sci. NS-37 (1990). To be published in these proceedings.

[8] W.W. Moses and S.E. Derenzo. Cerium Fluoride, a new fast, heavy scintillator. IEEE Trans. Nucl. Sci. NS-36, 173-176 (1989).

[9] D.F. Anderson. Properties of the high-density scintillator cerium fluoride. IEEE Trans. Nucl. Sci. NS-36, $137-140$ (1989).

[10] W.W. Moses and S.E. Derenzo. Lead Carbonate, a new fast, heavy scintillator. IEEE Trans. Nucl. Sci. NS-37 (1990). To be published in these proceedings. 


\section{DISCLAIMER}

This document was prepared as an account of work sponsored by the United States Government. Neither the United States Govemment nor any agency thereof, nor The Regents of the University of California, nor any of their employees, makes any warranty, express or implied, or assumes any legal liability or responsibility for the accuracy, completeness, or usefulness of any information, apparatus, product, or process disclosed, or represents that its use would not infringe privately owned rights. Reference herein to any specific commercial products process, or service by its trade name, trademark, manufacturer, or otherwise, does not necessarily constitute or imply its endorsement. recommendation, or favoring by the United States Government or any agency thereof, or The Regents of the University of California. The views and opinions of authors expressed herein do not necessarily state or reflect those of the United States Government or any agency thereof or The Regents of the University oi Califormia and shall not be used for advertising or product endorsement purposes.

Lawrence Berkejey Laboratory is an equal opportunity employer. 